\title{
Non-Perfect-Fluid Space-Times in Thermodynamic Equilibrium and Generalized Friedmann Equations
}

\author{
Konrad Schatz, Horst-Heino von Borzeszkowski, and Thoralf Chrobok \\ Institut für Theoretische Physik, Technische Universität Berlin, Hardenbergstraße 36, 10623 Berlin, Germany \\ Correspondence should be addressed to Konrad Schatz; konrads@justmail.de
}

Received 9 June 2016; Revised 29 August 2016; Accepted 13 October 2016

Academic Editor: Sergei D. Odintsov

Copyright (C) 2016 Konrad Schatz et al. This is an open access article distributed under the Creative Commons Attribution License, which permits unrestricted use, distribution, and reproduction in any medium, provided the original work is properly cited.

\begin{abstract}
We determine the energy-momentum tensor of nonperfect fluids in thermodynamic equilibrium and, respectively, near to it. To this end, we derive the constitutive equations for energy density and isotropic and anisotropic pressure as well as for heat-flux from the corresponding propagation equations and by drawing on Einstein's equations. Following Obukhov on this, we assume the corresponding space-times to be conform-stationary and homogeneous. This procedure provides these quantities in closed form, that is, in terms of the structure constants of the three-dimensional isometry group of homogeneity and, respectively, in terms of the kinematical quantities expansion, rotation, and acceleration. In particular, we find a generalized form of the Friedmann equations. As special cases we recover Friedmann and Gödel models as well as nontilted Bianchi solutions with anisotropic pressure. All of our results are derived without assuming any equations of state or other specific thermodynamic conditions a priori. For the considered models, results in literature are generalized to rotating fluids with dissipative fluxes.
\end{abstract}

\section{Introduction}

In this paper, we consider systems described by Einstein's equations:

$$
R_{a b}-\frac{1}{2} R g_{a b}=T_{a b}
$$

with an energy-momentum tensor and equations of state, neither of which are specified by any ad hoc assumptions. Instead, we discuss the whole question from a thermodynamic perspective (we emphasize that we approach this without any specific thermodynamic conditions as done, e.g., in [1]; i.e., we refrain from applying linear or extended thermodynamics). This consideration is discussed for a class of cosmologically interesting metrics (introduced and shown to be observationally admissible in [2-5], see (5) below). In terms of the temperature and the kinematic invariants characterizing the matter, our consideration provides a class of general equations of state ("matter equations") which are compatible with Einstein's equations and correspond to generalized Friedmann equations. This framework can find (and has found) applications in relativistic cosmology and astrophysics. Basically, it allows to go beyond the standard phase cosmology (governed by phases with certain equations of state like inflation, radiation, and dust that have to be fitted by fine-tuning) and to describe the cosmological state transitions from phase to phase by intermediate stages. However, in [6-9] a fine-tuned sudden passage from the decelerated to the accelerated regime, as observed today, produces inadequacies. These are then avoided by ad hoc introduced equations of state where viscosity originates from geometry (e.g., $H, \dot{H})$. Our calculations can provide a theoretical foundation of such equations. Furthermore, this framework also contributes to a physical discussion of no-go theorems like the shear-free fluid conjecture [10]. For instance, this thermodynamic approach enables one to sharpen the theorem (proved in [11], without explicitly referring to thermodynamics) which states for nonvanishing acceleration that rotation and expansion cannot simultaneously be equal to zero: in [11] it has been shown that models with vanishing acceleration do not allow for nonvanishing rotation.

Ehlers et al. have proven [12] that the high isotropy of the cosmic microwave background (CMB) and the vanishing of shear $\sigma_{a b}$ of a congruence of curves (for the definition of the quantities, shear, rotation, acceleration, and expansion, see [1] or see also Section 2) are closely related to the requirement 
that there exists a conformal Killing vector field (in [13], it is shown that while for $\sigma_{a b}=0$ fluids the CKV property is essential, for $\sigma_{a b} \neq 0$ fluids this property has to be generalized to conformal collineation) being parallel to the tangents of the curves (i.e., to the velocity of a streaming fluid). In detail, it was shown (Lemma 3 in [12]) that a space-time admits a timelike conformal Killing vector field (CKV)

$$
\xi_{a ; b}+\xi_{b ; a}=\Phi g_{a b}
$$

with $\xi_{a}=\alpha u_{a}$, if and only if there is a velocity field $u_{a}$ with $u_{a} u^{a}=-1$ satisfying

$$
\begin{aligned}
\sigma_{a b} & =0, \\
\left(\dot{u}_{[a}-\frac{\Theta}{3} u_{[a}\right)_{; b]} & =0,
\end{aligned}
$$

where $\dot{u}_{a}$ is the acceleration and $\Theta$ the expansion. Oliver Jr. and Davis [14] showed that (3) is a necessary and sufficient condition for the existence of a CKV in the case of rotating space-times, too. In the following, we consider such conformstationary space-times (according to $[15,16]$ this is equivalent to parallax-free cosmological models).

In particular, the second condition allows implying a parameter $\alpha$ which can be identified as the inverse temperature, $\alpha=1 / T$, so that $\xi_{a}=u_{a} / T$ can be interpreted as temperature vector. This parameter occurs if the second equation in (3) is rewritten as (Theorem 2.1 in [14])

$$
\frac{\alpha_{, a}}{\alpha}=\dot{u}_{a}-\frac{\Theta}{3} u_{a}
$$

Additionally, we assume that the considered spacetimes are spatially homogeneous. This reduction to Bianchitype models still allows for the matter distribution to be anisotropic, while the $\mathrm{CMB}$ is isotropic.

Altogether we are led to the subclass of tilted Bianchi models constructed by Obukhov [2-5] that admit a CKV,

$$
\begin{aligned}
d s^{2} & =g_{a b} d x^{a} d x^{b} \\
& =-d t^{2}+2 a n_{\widehat{a}} d x^{\widehat{a}} d t+a^{2} \gamma_{\widehat{a} \widehat{b}} d x^{\widehat{a}} d x^{\widehat{b}} .
\end{aligned}
$$

Thereby, rotating and expanding models with acceleration and isotropic CMB are considered in [2-5]. In contrast to that, in [17-19], Ehlers-Geren-Sachs theorems were, partly in a generalized version, used to study and determine a class of space-times containing also inhomogeneous cosmological models, with nontrivial acceleration but zero rotation.

To complete notation used in (5), we define

$$
\begin{aligned}
n_{\widehat{a}} & =\nu_{\widehat{\mu}} e^{\widehat{\mu}}, \\
\gamma_{\widehat{a} \hat{b}} & =\beta_{\widehat{\mu} \hat{\nu}} e^{\widehat{\mu}}{ }_{\widehat{a}} e^{\widehat{v}}
\end{aligned}
$$

with $\nu_{\widehat{\mu}}$ and $\beta_{\widehat{\mu} \widehat{\nu}}$ as arbitrary constants. Here the triad components $e^{\widehat{\mu}}{ }_{\widehat{a}}=e^{\widehat{\mu}}{ }_{\hat{a}}\left(x^{\widehat{k}}\right)$ form a basis which is invariant under the spatial isometries of the Bianchi models. Accordingly, their Lie derivative with respect to the generating Killing vector fields (KV) vanishes (for details see [20]). The components $e^{\widehat{\mu}}{ }_{\widehat{a}}\left(x^{\widehat{k}}\right)$ are supposed to be functions of the space-like canonic coordinates only and determine the metric (5) as to the Bianchi-type. The coordinate $t=x^{0}$ denotes the proper time with respect to a fluid particle and $a=a(t)$ is the scale factor.

Furthermore, Latin indices are used for the coordinate while Greek indices are used for the tetrad description of the tensor components. All indices with hat denote the three spatial dimensions, for example, $\widehat{a}=1,2,3$ and $\widehat{\alpha}=$ (1), (2), (3), whereas those without hat run through all four space-time dimensions, $a=0,1,2,3$ and $\alpha=(0),(1),(2),(3)$.

Regarding the thermodynamic proposition, we follow the Eckart approach and assume that the model under consideration is in thermodynamic (near)equilibrium [21]. The Eckart approach to the relativistic Theory of Irreversible Processes [22] (see also [23-25]) is based on the balance equations for the particle number (where $\mu$ represents mass density and $v=(1 / \mu)$ the specific volume $)$

$$
\left(\mu u^{a}\right)_{; a}=0
$$

the energy-momentum

$$
T_{; b}^{a b}=0
$$

(in particular, regarding (7), the null-component can be interpreted as the first law of thermodynamics [26, 27], i.e., as the conservation of internal energy), and the entropy

$$
\sigma=s_{; a}^{a} \geq 0
$$

where $s^{a}$ denotes the entropy vector and $\sigma$ the density of the nonnegative entropy production. In the case of thermodynamic equilibrium (vanishing of entropy production), appropriated supplementary conditions have to be added by hand. In the general-relativistic version of this theory the framework is completed by Einstein's gravitational equations (1). However, in the general-relativistic Theory of Irreversible Processes no further assumptions have to be introduced ad hoc in order to yield thermodynamic equilibrium [21]. 28],

Now, if the entropy vector is defined according to $[23,24$,

$$
s^{a}=\mu s u^{a}+\frac{q^{a}}{T}
$$

the entropy production can be reformulated as

$$
\sigma=\frac{u_{b}}{T}\left(T^{a b}-\rho u^{a} u^{b}-p h^{a b}\right)_{; a}+\left(\frac{q^{a}}{T}\right)_{; a} .
$$

Here $q^{a}$ denotes the heat-flux, $\rho$ the energy density, $p$ the pressure, and $u^{a} / T$ the temperature vector and $h^{a b}=g^{a b}+$ $u^{a} u^{b}$. Finally, by decomposing the energy-momentum tensor (27) this yields [28]

$$
\sigma=-\left(\frac{u_{b}}{T}\right)_{; a}\left(T^{a b}-\rho u^{a} u^{b}-p h^{a b}\right) .
$$


As shown in [21], regarding the conformal Killing equation (2), the second term in brackets turns out to be traceless which results in a vanishing entropy production $\sigma$

$$
\left(T^{a b}-\rho u^{a} u^{b}-p h^{a b}\right)\left(\xi_{a ; b}+\xi_{b ; a}\right)=0
$$

This shows that nonperfect fluids are not necessarily incompatible with reversible thermodynamics [28-30]. However, for space-times without a CKV or KV (13) can only be solved by assuming a perfect fluid. That this CKV property is not purely mathematical but has also a physical meaning is supported by the following arguments.

Firstly, the derivations of (12) and (13), given in [28], show that the quantities $\rho, p$, and $T$ are thermodynamically well determined. Indeed, it is assumed that the specific entropy $s$ is given as a function of the specific internal energy $u$ and the specific volume $v$; that is,

$$
s=s(u, v),
$$

so that (Gibbs equation)

$$
\begin{aligned}
T d s & =d u+p d v, \\
\frac{\partial s}{\partial u} & =\frac{1}{T} \\
\frac{\partial s}{\partial v} & =\frac{p}{T} .
\end{aligned}
$$

Moreover, for a comoving observer the relation $\rho=\mu(1+$ $u$ ) holds. For the thermodynamic quantities defined in this way (13) is valid. Secondly, (13) has the following solutions: Either the fluid is perfect or the temperature vector has to be a CKV (containing the special case of a Killing vector field). Therefore, the CKV property is justified by defining equilibrium or near-equilibrium states in the framework of reversible thermodynamics [21].

This is confirmed by the fact that $\xi^{a}=u^{a} / T$, being a $\mathrm{CKV}$, leads to some well-known models like Friedmann's and Gödel's space-times with the corresponding equations of state (see Section 3.2). Furthermore, it should be emphasized that the justification of the thermodynamic meaning of the CKV condition given via (13), that is, in the context of phenomenological continuum theory, is supported by considerations in the framework of kinetic theory, where the CKV property of $\xi^{a}$ in combination with related equations of state for some special cases is derived from Boltzmann's equation [31-34].

Based on the existence of such a CKV one can derive a set of four propagation equations for nonperfect fluids (see [11]), which link the propagation of the matter content to the kinematic description of the space-time (see Section 3).

The paper is organized as follows: In Section 2, we introduce a suitable tetrad frame that allows us to establish manageable equations. In addition, the decomposition of the energy-momentum tensor with respect to kinematic invariants is shortly reviewed and their form in tetrads for the space-times (5) is derived. Subsequently, by solving the propagation equations, we deduce in Section 3 general expressions for the whole matter content depending on the structure constants and the kinematic invariants, respectively. After checking the consistency with Einstein's equations the general case of a nonperfect fluid and particular cases like nontilted [35] and stationary models are discussed. Among the special cases that can be recovered are the Friedmann and the Gödel models. In Section 4 we discuss the results and provide alternative formulations, relevant for further observational and thermodynamic considerations.

\section{Tetrad Formulation and Kinematic Invariants}

In the following, we introduce tetrads (see, e.g., [36]) that allow for a convenient separation of the variable objects, $a(t)$ and $e^{\widehat{\mu}}{ }_{\widehat{a}}^{\widehat{k}}\left(x^{\widehat{k}}\right)$, and the constants, $\nu_{\widehat{\mu}}$ and $\beta_{\widehat{\mu} \widehat{\nu}}$ in (5). Defining

$$
\begin{aligned}
& \widehat{e}^{\alpha}{ }_{b}:=\left(\begin{array}{cc}
0 & 0 \\
0 & e^{\widehat{\alpha}}
\end{array}\right), \\
& \check{e}_{\alpha}{ }^{b}:=\left(\begin{array}{cc}
0 & 0 \\
0 & e_{\widehat{\alpha}}^{\widehat{b}}
\end{array}\right)
\end{aligned}
$$

with

$$
\check{e}_{\alpha}{ }^{b} \widehat{e}_{b}^{\beta}=\left(\begin{array}{cc}
0 & 0 \\
0 & \delta_{\widehat{\alpha}}^{\beta}
\end{array}\right),
$$

the tetrads can be chosen as

$$
\begin{aligned}
& \theta_{b}^{\alpha}=\delta_{0}^{\alpha} \delta_{b}^{0}+a \widehat{e}_{b}^{\alpha}, \\
& \theta_{\alpha}{ }^{b}=\delta_{\alpha}^{0} \delta_{0}^{b}+a^{-1} \check{e}_{\alpha}{ }^{b} .
\end{aligned}
$$

To fulfill the relations

$$
g_{a b}=\zeta_{\mu \nu} \theta_{a}^{\mu} \theta_{b}^{\nu}
$$

the constant and symmetric matrix $\zeta_{\mu \nu}$ has to take the form

$$
\zeta_{\mu \nu}=\left(\begin{array}{cc}
-1 & \nu_{\widehat{\nu}} \\
\nu_{\widehat{\mu}} & \beta_{\widehat{\mu} \widehat{\nu}}
\end{array}\right) .
$$

The structure constants of the isometry groups acting on the space-like hypersurfaces and specific to the Bianchi models can be expressed by a 4-dimensional representation:

$$
\widehat{C}_{\beta \alpha}^{\gamma}:=2 \check{e}_{[\alpha}^{b}{ }^{b} \widehat{e}_{b, c \mid}^{\gamma} \check{e}_{\beta]}^{c},
$$

such that $\widehat{C}_{0 \alpha}^{\gamma}=0, \widehat{C}_{\beta 0}^{\gamma}=0$, and $\widehat{C}_{\beta \alpha}^{0}=0$. Expressions for the curvature tensors and scalars in terms of these newly introduced tetrads are derived in Appendix B.

On the basis of these preliminaries, we now introduce the kinematic invariants and the respective decomposition of the energy-momentum tensor.

Assuming a one-component fluid with the four-velocity $u^{a}$, such that $u_{a} u^{a}=-1$, the gradient of $u^{a}$ can be decomposed kinematically [1]:

$$
u_{a ; b}=\omega_{a b}+\sigma_{a b}+\frac{\Theta}{3} h_{a b}-\dot{u}_{a} u_{b}
$$


Thus, rotation, shear, acceleration, and expansion as well as the scalar quantities of rotation and acceleration read

$$
\begin{aligned}
\omega_{a b} & =h_{a}^{c} h_{b}^{d} u_{[c ; d]}=u_{[a ; b]}+\dot{u}_{[a} u_{b]}, \\
\sigma_{a b} & =h_{a}^{c} h_{b}^{d} u_{(c ; d)}-\frac{\Theta}{3} h_{a b}, \\
\dot{u}_{a} & =u_{a ; b} u^{b}, \\
\Theta & =u_{; a}^{a}, \\
\omega^{2} & =\left(\frac{1}{2}\right) \omega_{\alpha \beta} \omega^{\alpha \beta}, \\
\dot{u}^{2} & =\dot{u}_{\alpha} .
\end{aligned}
$$

Choosing $u^{a}=\delta_{0}^{a}$, these kinematic quantities are rewritten in the tetrad representation and for the space-times (5) as follows (the subscript $\|$ denotes the covariant tetrad derivative)

$$
\begin{aligned}
\omega_{\alpha \beta} & =u_{[\alpha \| \beta]}+\dot{u}_{[\alpha} u_{\beta]}=\frac{1}{2 a} \widehat{C}_{\beta \alpha}^{\gamma} \zeta_{\gamma 0}, \\
\dot{u}_{\alpha} & =u_{\alpha \| \rho} u^{\rho}=\frac{\dot{a}}{a} h_{\alpha}^{0}, \\
\Theta & =u^{\mu}{ }_{\| \mu}=\Omega_{\mu}{ }^{\mu}{ }_{0}=3 \frac{\dot{a}}{a},
\end{aligned}
$$

where $u_{\alpha}=\zeta_{\alpha 0}$.

According to [1], the energy-momentum tensor can be decomposed with respect to the timelike velocity field $u_{a}$ :

$$
T_{a b}=\rho u_{a} u_{b}+p h_{a b}+2 u_{(a} q_{b)}+\pi_{a b} .
$$

Here the quantities can be identified with the appropriate projections, $\rho=T_{a b} u^{a} u^{b}$ for the energy density, $p=(1 / 3) T_{a b} h^{a b}$ for the isotropic pressure, $q_{a}=-T_{c b} u^{b} h_{a}^{c}$ for the heat-flux, and $\pi_{a b}=T_{c d} h_{a}^{c} h_{b}^{d}-p h_{a b}$ for the anisotropic pressure.

\section{Matter Equations}

The conditions for the temperature vector $u_{b} T^{-1}$, being a $\mathrm{CKV}$, are

$$
\begin{aligned}
& \Phi=\frac{2}{3} \frac{\Theta}{T}, \\
& \Theta=3 T\left(\frac{1}{T}\right)_{, 0} .
\end{aligned}
$$

Both can be found in [14] (the latter reproduces the 0component of (4)). The two results (28) and (29) are obtained by inserting $u_{b} T^{-1}$ into (2) and multiplying this equation by $u^{a} u^{b}$ and $g^{a b}$, respectively.

Furthermore, integration of (29) leads to an expression for the temperature scalar,

$$
T=\frac{1}{{ }_{T} c a}
$$

and the conformal factor,

$$
\Phi=2_{T} c \dot{a},
$$

where ${ }_{T} \mathcal{C}$ is the constant of integration.
The existence of the CKV has far-reaching consequences for the geometry of the space-time and, factoring in Einstein's field equations, for the matter. By drawing on the Ricci identity for the CKV and the Bianchi identity subsequently, we deduce a set of four propagation equations [11, 21]. The first two describe the evolution of the energy density $\rho$ and the isotropic pressure $p$ :

$$
\begin{aligned}
& -\frac{1}{2} \square \Phi+\ddot{\Phi}-\Phi_{; m} \dot{u}^{m}+\frac{1}{2 T}(3 \dot{p}+\dot{\rho})+\frac{\Theta(3 p+\rho)}{3 T} \\
& \quad=0, \\
& 3 \square \Phi-\frac{(3 \dot{p}-\dot{\rho})}{T}-\frac{2 \Theta(3 p-\rho)}{3 T}=0 .
\end{aligned}
$$

The other two equations describe the change of the heat-flux $q_{a}$,

$$
\begin{aligned}
h_{a}^{b} \dot{q}_{b}= & T \dot{\Phi}_{, b} h_{a}^{b}-T \Phi_{, m} \omega_{a}^{m}-\frac{1}{3} T \Phi_{, m} \Theta h_{a}^{m}-\frac{2}{3} \Theta q_{a} \\
& -q^{k} \omega_{k a},
\end{aligned}
$$

and the anisotropic pressure $\pi_{a b}$,

$$
\begin{aligned}
h_{a}^{m} h_{c}^{b} \dot{\pi}_{b m}= & -\frac{T}{2} h_{a c} \square \Phi-T h_{a}^{m} h_{c}^{b} \Phi_{, m ; b}+h_{a c} \frac{\dot{p}-\dot{\rho}}{2} \\
& +2 \pi^{k}{ }_{(a} \omega_{c) k}-\frac{2 \Theta}{3} \pi_{a c}+\frac{\Theta(p-\rho)}{3} h_{a c} .
\end{aligned}
$$

3.1. Solutions of the Propagation Equations. The reformulation of the dynamic equations (32)-(34) in terms of the spacetimes (5) and some tedious algebra brings us to a set of ordinary differential equations which can be solved analytically.

To this end, we decouple (32) to

$$
\begin{aligned}
& \dot{\rho}=-\frac{2}{3} \Theta \rho-T \square \Phi-T \ddot{\Phi}+T \Phi_{, m} \dot{u}^{m}, \\
& \dot{p}=-\frac{2}{3} \Theta p+\frac{2}{3} T \square \Phi-\frac{1}{3} T \ddot{\Phi}+\frac{1}{3} T \Phi_{, m} \dot{u}^{m} .
\end{aligned}
$$

By means of (18), (35) can be rewritten as

$$
\begin{aligned}
& \left(a^{2} \rho\right)_{, 0}+2 \ddot{a} a\left(\zeta^{00}+1\right)+2 \ddot{a} \dot{a}\left(2 \zeta^{00}-1\right) \\
& +2 \ddot{a} \widehat{C}_{\kappa \gamma}^{\gamma} \zeta^{\kappa 0}=0, \\
& \left(a^{2} p\right)_{, 0}+\frac{2}{3} \ddot{a} a\left(1-2 \zeta^{00}\right)-\frac{2}{3} \ddot{a} \dot{a}\left(7 \zeta^{00}+1\right) \\
& -\frac{4}{3} \ddot{a} \widehat{C}_{\kappa \gamma}^{\gamma} \zeta^{\kappa 0}=0 .
\end{aligned}
$$


Integrating (36) yields

$$
\begin{aligned}
\rho= & -2\left(\frac{\dot{a}}{a}\right)_{, 0}\left(\zeta^{00}+1\right)-3\left(\frac{\dot{a}}{a}\right)^{2} \zeta^{00}-2 \frac{\dot{a}}{a^{2}} \widehat{C}_{\kappa \gamma}^{\gamma} \zeta^{\kappa 0} \\
& +\frac{1}{a^{2}} \rho^{c,} \\
p= & \frac{2}{3}\left(\frac{\dot{a}}{a}\right)_{, 0}\left(2 \zeta^{00}-1\right)+3\left(\frac{\dot{a}}{a}\right)^{2} \zeta^{00}+\frac{4}{3} \frac{\dot{a}}{a^{2}} \widehat{C}_{\kappa \gamma}^{\gamma} \zeta^{\kappa 0} \\
& +\frac{1}{a^{2}} p^{c,}
\end{aligned}
$$

where the objects ${ }_{\rho} c$ and ${ }_{p} c$ represent the summed constants of integration of the additive antiderivatives; see Appendix C. For concrete cosmological or astrophysical models, for example, stars, the boundaries of the respective integrals are specified.

With the identity

$$
h_{\mu}^{\rho}\left(\zeta^{\mu \kappa} \omega_{\alpha \kappa}+h_{\alpha}^{\nu} u^{\gamma} \Omega_{\gamma{ }_{\nu}}\right)=0
$$

and (24), the tetrad formulations for the propagation equations of the heat-flux (33) and the anisotropic pressure (34) together with the kinematic quantities $(24)-(26)$ yield the following integrable partial differential equations:

$$
\begin{aligned}
& \left(q_{\alpha} a^{2}\right)_{, 0}+2(\ddot{a} \dot{a}-\ddot{a} a) h_{\alpha}^{0}+\ddot{a} \widehat{C}^{\widehat{\gamma}}{ }_{\alpha \sigma} \zeta_{\widehat{\gamma} 0} \zeta^{\sigma 0}=0, \\
& 3\left(a^{2} \pi_{\alpha \gamma}\right)_{, 0}+2(\ddot{a} \dot{a}-\ddot{a} a)\left(h_{\alpha \gamma}\left(\zeta^{00}+1\right)-3 h_{\alpha}^{0} h_{\gamma}^{0}\right) \\
& \quad+2 \ddot{a} \widehat{C}_{\mu \kappa}^{\rho} \zeta^{\kappa 0}\left(\delta_{\rho}^{\mu} h_{\alpha \gamma}-3 \delta_{(\alpha}^{\mu} h_{\gamma) \rho}\right)=0
\end{aligned}
$$

for the heat-flux and the anisotropic pressure, respectively.

Integration and reorganization of terms bring the wanted solutions

$$
\begin{aligned}
q_{\alpha}= & 2\left(\frac{\dot{a}}{a}\right)_{, 0} h_{\alpha}^{0}+\frac{\dot{a}}{a^{2}} \widehat{C}_{\sigma \alpha}^{\gamma} \zeta_{\gamma 0} \zeta^{\sigma 0}+\frac{1}{a^{2}}{ }_{q} c_{\alpha}, \\
\pi_{\alpha \beta}= & \frac{2}{3}\left(\frac{\dot{a}}{a}\right)_{, 0} h_{\alpha}^{0}\left(h_{\alpha \beta}\left(\zeta^{00}+1\right)-3 h_{\alpha}^{0} h_{\beta}^{0}\right) \\
& +\frac{2}{3} \frac{\dot{a}}{a^{2}} \widehat{C}_{\kappa \mu}^{\rho} \zeta^{\kappa 0}\left(\delta_{\rho}^{\mu} h_{\alpha \beta}-3 \delta_{(\alpha}^{\mu} \zeta_{\beta) \rho}\right) \\
& +\frac{1}{a^{2}}{ }_{\pi} c_{\alpha \beta},
\end{aligned}
$$

where, similarly to the case of the energy density (37) and the isotropic pressure (38) above, the objects ${ }_{q} c_{\alpha}$ and ${ }_{\pi} c_{\alpha \gamma}$ represent the constants of integration (see Appendix C).

With the help of the kinematic quantities (24)-(26) and (A.1)-(A.4) of Appendix A the solutions (37), (38), (41), and (42) can be rewritten as follows:

$$
\begin{aligned}
& \rho=\frac{1}{3} \Theta^{2}+3 \dot{u}^{2}-2 \dot{u}^{\gamma}{ }_{\| \gamma}+T^{2}{ }_{T} c^{2}{ }_{\rho} c, \\
& p=-\frac{2}{3} \dot{\Theta}-\frac{1}{3} \Theta^{2}-\dot{u}^{2}+\frac{4}{3} \dot{u}^{\gamma}{ }_{\| \gamma}+T^{2}{ }_{T} c^{2}{ }_{p} c,
\end{aligned}
$$

$$
\begin{aligned}
q_{\alpha}= & \frac{2}{3} \dot{\Theta} h_{\alpha}^{0}+2 \omega_{\alpha \gamma} \dot{u}^{\gamma}+T_{T}^{2}{ }_{T}{ }^{2}{ }_{q} c_{\alpha}, \\
\pi_{\alpha \beta}= & -\frac{2}{3} \dot{\Theta} h_{\alpha}^{0} h_{\beta}^{0}-2 \dot{u}^{2} h_{\alpha \beta}+\frac{2}{3} \dot{u}^{\gamma}{ }_{\| \gamma} h_{\alpha \beta} \\
& -2 T \dot{u}^{\kappa} \widehat{C}^{\rho}{ }_{\kappa(\alpha} h_{\beta) \rho}+T^{2}{ }_{T} c^{2}{ }_{\pi} c_{\alpha \beta}
\end{aligned}
$$

or, in terms of purely kinematic quantities,

$$
\begin{aligned}
\pi_{\alpha \beta}= & \frac{2}{3} \dot{\Theta}\left(2 h_{(\alpha}^{0} u_{\beta)}-3 h_{\alpha}^{0} h_{\beta}^{0}\right)+\frac{2}{3}\left(\dot{u}^{\gamma}{ }_{\| \gamma}-9 \dot{u}^{2}\right) h_{\alpha \beta} \\
& +4 \dot{u}_{(\alpha \| \beta)}-\frac{4}{3} \Theta \dot{u}_{(\alpha} u_{\beta)}+4 \omega_{\kappa(\alpha} u_{\beta)} \dot{u}^{\kappa}+4 \dot{u}_{\alpha} \dot{u}_{\beta} \\
& -4 \dot{u}^{2} u_{\alpha} u_{\beta}+T_{T}^{2} c^{2}{ }_{\pi} c_{\alpha \beta} .
\end{aligned}
$$

The expressions (43) and (44) can be understood as generalized Friedmann equations.

According to (27), we can now reconstruct the energymomentum tensor by inserting the four solutions above:

$$
\begin{aligned}
T_{\alpha \beta}= & -\frac{2}{3} \dot{\Theta}\left(\zeta_{\alpha \beta}+\delta_{\alpha}^{0} \delta_{\beta}^{0}\right)+\frac{1}{3}\left(6 \dot{u}^{\gamma}{ }_{\| \gamma}-\Theta^{2}-9 \dot{u}^{2}\right) \zeta_{\alpha \beta} \\
& -2 T_{T} c \dot{u}^{\kappa} \widehat{C}^{\rho}{ }_{\kappa(\alpha} \zeta_{\beta) \rho}+T^{2}{ }_{T} c^{2}{ }_{E I} c_{\alpha \beta},
\end{aligned}
$$

or

$$
\begin{aligned}
T_{\alpha \beta}= & \frac{2}{3} \dot{\Theta}\left(2 h_{(\alpha}^{0} u_{\beta)}-3 h_{(\alpha}^{0} h_{\beta)}^{0}-h_{\alpha \beta}\right) \\
& -\dot{u}^{2}\left(7 h_{\alpha \beta}+u_{\alpha} u_{\alpha}\right)+\frac{1}{3}\left(\dot{u}^{\gamma}{ }_{\| \gamma}-\Theta^{2}\right) \zeta_{\alpha \beta} \\
& +4\left(\dot{u}_{(\alpha \| \beta)}+\dot{u}_{\alpha} \dot{u}_{\beta}\right)+T_{T}^{2}{ }_{T}{ }^{2}{ }_{E I} c_{\alpha \beta},
\end{aligned}
$$

where

$$
{ }_{E I} c_{\alpha \beta}={ }_{\rho} c \zeta_{\alpha 0} \zeta_{\beta 0}+{ }_{p} c h_{\alpha \beta}+2{ }_{q} c_{(\alpha} \zeta_{\beta) 0}+{ }_{\pi} c_{\alpha \beta} .
$$

In order to verify the consistency of solutions (43)-(46) with Einstein's field equations and in order to recover special cases, the constants of integration are determined by the calculations of Appendix C. The matter equations (43)-(46) then take the exclusively kinematic forms:

$$
\begin{aligned}
\rho= & \frac{1}{3} \Theta^{2}+3 \dot{u}^{2}-2 \dot{u}_{\| \gamma}^{\gamma}-\frac{1}{2} \widetilde{R} T_{T}^{2} c^{2}+2 \omega^{2}, \\
p= & -\frac{2}{3} \dot{\Theta}-\frac{1}{3} \Theta^{2}-\dot{u}^{2}+\frac{4}{3} \dot{u}_{\| \gamma}^{\gamma}+\frac{1}{6} \widetilde{R} T_{T}^{2} c^{2}+\frac{2}{3} \omega^{2}, \\
q_{\alpha}= & \frac{2}{3} \dot{\Theta} h_{\alpha}^{0}+\omega_{\alpha \gamma} \dot{u}^{\gamma}+\omega^{\tau \gamma}{ }_{\| \gamma} h_{\tau \alpha}, \\
\pi_{\alpha \beta}= & \frac{2}{3} \dot{\Theta}\left(2 h_{(\alpha}^{0} u_{\beta)}-3 h_{\alpha}^{0} h_{\beta}^{0}\right)-2\left(2 \dot{u}^{2}+\omega^{2}\right) u_{\alpha} u_{\beta} \\
& +\frac{1}{3}\left(2 \dot{u}_{\| \gamma}^{\gamma}-18 \dot{u}^{2}-2 \omega^{2}+\widetilde{R} T_{T}^{2} c^{2}\right) h_{\alpha \beta} \\
& -\widetilde{R}_{\alpha \beta} T_{T}^{2} c^{2}-\frac{4}{3} \dot{u}_{(\alpha} u_{\beta)}+4 \dot{u}_{(\alpha \| \beta)}+4 \dot{u}_{\alpha} \dot{u}_{\beta} \\
& +2 \omega_{\kappa(\alpha} u_{\beta)} \dot{u}^{\kappa}-2 \omega^{\tau \mu}{ }_{\| \mu} h_{\tau(\alpha} u_{\beta)},
\end{aligned}
$$


in which for the latter, $\pi_{\alpha \beta}$, relation (A.3) was used in addition. By multiplying these expressions with the tetrads, one obtains the coordinate representation without any additional terms.

Notice that (51) and (52) satisfy the Raychaudhuri equation:

$$
\rho+3 p=-2 \dot{\Theta}-\frac{2}{3} \Theta^{2}+4 \omega^{2}+2 \dot{u}_{; a}^{a} .
$$

\subsection{Special Cases}

3.2.1. Nontilted Models. The nontilted limit $\left(h_{\alpha}^{0}=0\right)$ leads to purely expanding models, that is, those with vanishing rotation and acceleration. In this case also the coefficients $n_{\widehat{a}}$ of (5) become zero, so that the space-times are conformally static $[37,38]$.

Following up on this premise, (51) and (52) turn into the equations

$$
\begin{aligned}
& \rho=3\left(\frac{\dot{a}}{a}\right)^{2}-\frac{\widetilde{R}}{2 a^{2}}, \\
& p=-\left(\frac{\dot{a}}{a}\right)^{2}-2 \frac{\ddot{a}}{a}+\frac{\widetilde{R}}{6 a^{2}} .
\end{aligned}
$$

These correspond to the Friedmann equations

$$
\begin{aligned}
& \frac{1}{3} \rho=\left(\frac{\dot{a}}{a}\right)^{2}+\frac{k}{a^{2}}, \\
& -p=2 \frac{\ddot{a}}{a}+\left(\frac{\dot{a}}{a}\right)^{2}+\frac{k}{a^{2}},
\end{aligned}
$$

if the curvature parameter $k$ and the Ricci scalar $\widetilde{R}$ of the 3 dimensional Bianchi spaces are related by

$$
k=-\frac{1}{6} \widetilde{R} .
$$

This result is in accordance with [39, p. 474]. The two constants of integration are then related by

$$
p^{c}=-\frac{1}{3} \rho^{c}=\frac{1}{6} \widetilde{R}
$$

Furthermore, the heat-flux (53) is identically zero, while for the anisotropic pressure (54) one gets

$$
\pi_{\alpha \beta}={ }_{\pi} c_{\alpha \beta} T_{T}^{2} c^{2}=-\widetilde{R}_{\alpha \beta} T_{T}^{2} c^{2}+\frac{1}{3} \widetilde{R} T_{T}^{2} c^{2} h_{\alpha \beta} .
$$

According to Section 4 of [37], the nonperfect fluid models investigated here can be subdivided into three further classes. In detail, this amounts to determining the number of distinct eigenvalues of the anisotropic pressure (60): If $\pi_{\alpha \beta}$ has three different eigenvalues, the space-time is of Petrov-type I. In the case of two different eigenvalues, one obtains Petrov-type D. Finally, if there is only one eigenvalue, it can only be zero and results in $\pi_{\alpha \beta}$ vanishing identically. Therefore, only this latter case is in general a sufficient condition for obtaining perfect fluid Friedmann models.
An example for a nontilted space-time subclass of (5) which does not contain Friedmann models is provided by the Bianchi-type IV metric

$$
\begin{aligned}
d s^{2} & =-d t^{2}+a^{2}\left(d x^{2} \beta_{11}+2 d x e^{x}\left(d z \beta_{13}\right.\right. \\
& \left.+d y\left(\beta_{12}+x \beta_{13}\right)\right)+e^{2 x}\left(d z^{2} \beta_{33}\right. \\
& +2 d y d z\left(\beta_{23}+x \beta_{33}\right) \\
& \left.\left.+d y^{2}\left(\beta_{22}+2 x \beta_{23}+x^{2} \beta_{33}\right)\right)\right)
\end{aligned}
$$

with canonic coordinates $\left(x^{0} \rightarrow t, x^{1} \rightarrow x, x^{2} \rightarrow y, x^{3} \rightarrow z\right)$ and undetermined constants $\beta_{\widehat{a} \widehat{b}}$ introduced in (5). Since here one finds three distinct eigenvalues for (60), models (61) are of Petrov-type I.

For the particular case $\beta_{\widehat{a} \widehat{b}}=\mathbb{1}$, metric (61) takes the form

$$
\begin{aligned}
& d s^{2} \\
& =-d t^{2} \\
& \quad+a^{2}\left(d x^{2}+e^{2 x}\left(d z^{2}+2 x d y d z+d y^{2}\left(1+x^{2}\right)\right)\right)
\end{aligned}
$$

which coincides exactly with the example discussed in [37] (see (4.9) therein).

3.2.2. Stationary Models. We check the consistency of our results with the stationary limit as to $[3,4]$. Accordingly, for vanishing expansion and generally nontrivial rotation, (51) and (52) reduce to

$$
\begin{aligned}
& \rho={ }_{\rho}{ }^{{ }_{T} c^{2} T^{2}}=-\frac{1}{2} \widetilde{R} T_{T}^{2} c^{2}+2 \omega^{2}, \\
& p={ }_{p}{ }^{c_{T} c^{2} T^{2}}=\frac{1}{6} \widetilde{R} T_{T}^{2}{ }_{T} c^{2}+\frac{2}{3} \omega^{2},
\end{aligned}
$$

so that

$$
p=\frac{p^{c}}{{ }_{\rho} c} \rho=\text { const. }
$$

In the perfect fluid limit with vanishing heat-flux and anisotropic pressure, one obtains from (53) the condition

$$
q_{\alpha}={ }_{q} c_{\alpha T} c^{2} T^{2}=\omega^{\kappa \mu}{ }_{\| \mu} h_{\alpha \kappa}=0
$$

and from (54)

$$
\begin{aligned}
\pi_{\alpha \beta}= & { }_{\pi} c_{\alpha \beta T} c^{2} T^{2} \\
= & -\widetilde{R}_{\alpha \beta} T^{2}{ }_{T} c^{2}+\frac{1}{3} \widetilde{R} T_{T}^{2}{ }_{T}{ }^{2} h_{\alpha \beta} \\
& -\frac{2}{3} \omega^{2}\left(3 u_{\alpha} u_{\beta}+h_{\alpha \beta}\right)-2 \omega^{\tau \mu}{ }_{\| \mu} h_{\tau(\alpha} u_{\beta)}=0 .
\end{aligned}
$$

As a more concrete ansatz we choose a Bianchi-type III subclass of space-times (5):

$$
\begin{aligned}
(d s)^{2}= & (d t)^{2}-2 \sqrt{\Sigma} a e^{M x^{1}} d t d x^{2} \\
& -a^{2}\left(\left(d x^{1}\right)^{2}+K e^{2 M x^{1}}\left(d x^{2}\right)^{2}+\left(d x^{3}\right)^{2}\right),
\end{aligned}
$$


where $\nu_{\widehat{a}}=(0, \sqrt{\Sigma}, 0)$ and $\beta_{\widehat{a} \widehat{b}}=\operatorname{diag}(1, K, 1)$ and $e^{\widehat{\mu}}=$ $\operatorname{diag}\left(1, e^{M x^{1}}, 1\right)$ with $K, M$, and $\Sigma$ being constant. Admitting in general nonvanishing rotation and expansion, this metric is also denoted as the Gödel-type model (see [3, 4]). By this choice, the heat-flux (65) vanishes identically, while the anisotropic pressure condition (66) holds only for at least either of the two relations:

$$
K=-\frac{\Sigma}{2}
$$

or $M=0$.

Furthermore, one has

$$
\begin{aligned}
\widetilde{R} & =\frac{M^{2}(4 K+3 \Sigma)}{2(K+\Sigma)}, \\
\omega^{2} & =\frac{M^{2} \Sigma}{4 a^{2}(K+\Sigma)}
\end{aligned}
$$

or, by (68), respectively,

$$
\begin{aligned}
\widetilde{R} & =M^{2}, \\
\omega^{2} & =\frac{M^{2}}{2 a^{2}} .
\end{aligned}
$$

This yields ${ }_{p} c={ }_{\rho} c$ and thus, for expression (64),

$$
p=\rho=\frac{1}{2} \widetilde{R} T_{T}^{2}{ }_{T} c^{2}=\omega^{2} .
$$

According to, for example, [28], this is just the equation of state of the classical Gödel space-time. Indeed, in [3] it is stated that $K=-(1 / 2) \Sigma$ yields closed timelike curves.

\section{Discussion}

In this paper, we considered homogeneous and conform-stationary space-times (5) with Bianchi group invariance and an arbitrary matter source, which allows for generally tilted models.

By solving the propagation equations (32)-(34), we deduce explicit expressions for the energy density (37), the isotropic pressure (38), the heat-flux (41), and the anisotropic pressure (42) in terms of the scale factor, the tetrad components (18), and the structure constants. These results are rewritten in terms of the kinematic quantities, as to be found in (43), (44), (45), and (47), and are combined to the energymomentum tensor, (48) or (49). Similar equations are ad hoc assumed in [6-9] in order to solve problems arising during cosmological evolution for different reasons.

In addition to the Raychaudhuri equation and the other propagation and constraint equations (see, e.g., [1, 40]), we obtain equations in which the expressions for the matter content are decoupled and independent of higher derivatives of the kinematic quantities (except for the expansion and acceleration) or depending on the electric part of the Weyl tensor. Particularly, no equations of state or further thermodynamic relations have to be assumed to arrive at these results. Here it should be emphasized that the vanishing shear does not necessarily imply a zero anisotropic pressure as required by linear thermodynamics. It should also be pointed out that more-component fluids or a cosmological constant can easily be included.

Equations (37), (38), (41), and (42) represent a class of models which does not only contain physically relevant space-times. To take into account well-motivated (energy) conditions or global aspects (as considered in [41]) which should provide broader restrictions is therefore a subject of future research.

Moreover, inspection of (43), (44), (45), and (47) underlines that further thermodynamic assumptions like an equation of state, Fourier's law, Cauchy's law, or expressions from extended thermodynamics will further restrict possible solutions. This becomes manifest, if one rewrites (37), (38), (41), and (42) with the help of (29):

$$
\begin{aligned}
& \rho=-\left(\frac{\dot{T}}{T}\right)^{2}\left(5 \zeta^{00}+2\right)+2 \frac{\ddot{T}}{T}\left(\zeta^{00}+1\right) \\
& +2 \dot{T}_{T} c \widehat{C}_{\kappa \gamma}^{\gamma} \zeta^{\kappa 0}+T_{T}^{2} c_{\rho}^{2} c, \\
& p=\frac{1}{3}\left(\frac{\dot{T}}{T}\right)^{2}\left(13 \zeta^{00}-2\right)-\frac{2}{3} \frac{\ddot{T}}{T}\left(2 \zeta^{00}-1\right) \\
& -\frac{4}{3} \dot{T}_{T} c \widehat{C}^{\gamma}{ }_{\kappa \gamma} \zeta^{\kappa 0}+T_{T}^{2}{ }_{T}{ }^{2}{ }_{p} c, \\
& q_{\alpha}=2\left(\frac{\dot{T}^{2}-\ddot{T} T}{T^{2}}\right) h_{\alpha}^{0}-\dot{T}_{T} c \widehat{C}_{\sigma \alpha}^{\gamma} \zeta_{\gamma 0} \zeta^{\sigma 0} \\
& +T^{2}{ }_{T} c^{2}{ }_{q} c_{\alpha}, \\
& \pi_{\alpha \beta}=\frac{2}{3}\left(\frac{\dot{T}^{2}-\ddot{T} T}{T^{2}}\right)\left(h_{\alpha \beta}\left(\zeta^{00}+1\right)-3 h_{\alpha}^{0} h_{\beta}^{0}\right) \\
& -\frac{2}{3} \dot{T}_{T} c \widehat{C}_{\kappa \mu}^{\rho} \zeta^{\kappa 0}\left(\delta_{\rho}^{\mu} h_{\alpha \beta}-3 \delta_{(\alpha}^{\mu} \zeta_{\beta) \rho}\right) \\
& +T^{2}{ }_{T} c^{2}{ }_{\pi} c_{\alpha \beta} \text {. }
\end{aligned}
$$

These equations describe the temperature dependence of the matter content which has to be fulfilled for the considered class of models.

Expressions (72) and (73) can be used to construct equations of state. For instance, one can combine the two in such a way that the outcome does not contain the structure constants:

$$
\begin{aligned}
p+2 \rho= & 3\left(\frac{\dot{T}}{T}\right)^{2}\left(\zeta^{00}-2\right)+6 \frac{\ddot{T}}{T} \\
& +T_{T}^{2} c^{2}\left(2_{\rho} c+3{ }_{p} c\right)
\end{aligned}
$$

which is a possible equation of state for the considered spacetime class. This relation clearly shows that the pressure has a difficult dependence on the temperature and its first and second derivatives. Of course, $\rho$ has an explicit temperature 
dependence as given in (72), but assuming the validity of simple equations of state, like $p(\rho) \propto \rho^{\alpha}$, an effective finetuning has to be done in order to prevent an additional temperature dependence of $p$.

It becomes obvious from (74) and (75) that the assumption of Fourier's or Cauchy's laws consequently generates additional strong restrictions on the space-time and its matter content. The same is true for other ad hoc introduced constitutive equations. This includes nonlinear ones like the heat-flux law of Israel-Stewart-type [23] which is physically motivated by the fact that it overcomes stability and causality problems arising in the linear case. Our point, however, is to ask for those constitutive equations and equations of state, respectively, which follow from the conservation laws in a prescribed geometry and a given temperature field. Thus, it is not in the sense of the present consideration, to additionally impose ad hoc constitutive equations on (74) and (75). In nonrelativistic continuum thermodynamics the situation is different. There, one has to complete the system of basic equations resting on the conservation or balance equations by adding such ad hoc relations manually. If (74) and (75) differ from those ansatzes made by hand, this can have a variety of reasons and implications. To call only one, if there were severe thermodynamic arguments for one of the linear or nonlinear ad hoc ansatzes, for example, for the heat-flux, one was obliged to ask under which condition it is compatible with (74).

This view is reinforced by the results obtained in [42]. There, it was shown that in conform-stationary models the heat-flux must vanish for zero anisotropic pressure and under the assumption of a heat-flux law of the Israel-Stewart type. An example for physical processes in which this does not hold (Landau damping) is also provided in [42].

Moreover, the form of expressions (74) and (75), which denotes the modified laws of Fourier and Cauchy, is pointing in a direction that is to be found in various formulations of extended thermodynamics $[23,43]$. This becomes evident if one rewrites (75) with the help of (72) and (74):

$$
\begin{aligned}
\pi_{\alpha \beta} & \\
= & \frac{T^{2}}{2\left(\ddot{T} T-\dot{T}^{2}\right)}\left(q_{\alpha}+\dot{T}_{T} c \widehat{C}^{\gamma}{ }_{\sigma \alpha} \zeta_{\gamma 0} \zeta^{\sigma 0}-T_{T}^{2} c^{2}{ }_{q} c_{\alpha}\right) \\
& \cdot\left(q_{\beta}+\dot{T}_{T} c \widehat{C}^{\gamma}{ }_{\sigma \beta} \zeta_{\gamma 0} \zeta^{\sigma 0}-T^{2}{ }_{T} c^{2}{ }_{q} c_{\beta}\right) \\
& -h_{\alpha \beta}\left(\frac{1}{3} \rho+\left(\frac{\dot{T}}{T}\right)^{2} \zeta^{00}-\frac{1}{3} T^{2}{ }_{T} c^{2}{ }_{\rho} c\right) \\
& +2 \dot{T}_{T} c \widehat{C}^{\rho}{ }_{\kappa(\alpha} \zeta_{\beta) \rho} \zeta^{\kappa 0}+T^{2}{ }_{T} c^{2}{ }_{\pi} c_{\alpha \beta} .
\end{aligned}
$$

As a constitutive quantity $\pi_{\alpha \beta}$ is a function which is linear and quadratic in the heat-flow and linear in the energy density, while the temperature is also included with its first and second time derivative.

The consideration of simple models like nontilted or stationary ones leads back to, for example, the well-known Friedmann or the Gödel space-times (in both cases the constants of integration are determined; see Section 3) and similar anisotropic models as discussed in [37]. In this context expressions (43) and (44) or (51) and (52), respectively, can be understood as generalized Friedmann equations.

By rewriting (43), (44), (45), and (47) in terms of the observational quantities $H=\dot{a} / a$ for the Hubble function and $(\dot{a} / a)^{\circ}=-H^{2}(1+q)$ for the deceleration parameter $q$ one receives limits on acceleration, rotation, heat-flux, and anisotropic pressure.

The corresponding equations take the form

$$
\begin{aligned}
\rho= & \zeta^{00} H^{2}(2 q-1)+2 H^{2}(1+q)-2 H \frac{1}{a} \widehat{C}^{\gamma}{ }_{\kappa \gamma} \zeta^{\kappa 0} \\
& +\frac{1}{a^{2}} \rho^{c}, \\
p= & \frac{1}{3} \zeta^{00} H^{2}(5-4 q)+\frac{2}{3} H^{2}(1+q)+\frac{4}{3} \frac{H}{a} \widehat{C}^{\gamma}{ }_{\kappa \gamma} \zeta^{\kappa 0} \\
& +\frac{1}{a^{2}}{ }_{p}^{c}, \\
q_{\alpha}= & -2 H^{2}(1+q) h_{\alpha}^{0}+\frac{H}{a} \widehat{C}_{\kappa \alpha}^{\gamma} \zeta_{\gamma 0} \zeta^{\kappa 0}+\frac{1}{a^{2}}{ }_{q}^{c_{\alpha},} \\
\pi_{\alpha \beta}= & -\frac{2}{3} H^{2}(1+q)\left(h_{\alpha \beta}\left(\zeta^{00}+1\right)-3 h_{\alpha}^{0} h_{\beta}^{0}\right) \\
& +\frac{2}{3} \frac{H}{a} \widehat{C}^{\rho}{ }_{\kappa \mu} \zeta^{\kappa 0}\left(\delta_{\rho}^{\mu} h_{\alpha \beta}-3 \delta_{(\alpha}^{\mu} \zeta_{\beta) \rho}\right) \\
& +\frac{1}{a^{2}}{ }_{\pi} c_{\alpha \beta},
\end{aligned}
$$

so that the matter content can be described by the observable quantities $H$ and $q$ and the model-dependent constants $\zeta^{00}$, $h_{\alpha}^{0}$, and $\widehat{C}_{\beta \gamma}^{\alpha}$ as well as the constants of integration, eventually given by initial or boundary conditions.

In analogy to the calculations which lead to (76), one obtains from (78) and (79)

$$
2 \rho+p=3 H^{2}\left(2 q+2+\zeta^{00}\right)+\frac{1}{a^{2}}\left(2{ }_{\rho} c+3{ }_{p} c\right)
$$

which can again be regarded as an equation of state given by observational quantities. The class of models we consider here may have an anisotropic behavior of the Hubble flow and the galaxy distribution function $[3,4]$. In this context, the observation of a large-scale flow of galaxies, called "dark flow," with respect to the CMB is remarkable (see [44] for a review). A detailed discussion of this and other possibly observable effects in nonrotating models can be found in [17-19].

Refraining from possible further restrictions on relations (78)-(81), one finds the following hypothetical scenario.

For a large scale factor $a \gg 0$, the structure constants and the constants of integration are negligible, such that, for the behavior of the matter content, the expansion rate $H$ and the deceleration rate $q$ are most important. Moreover, one sees that $q$ has critical values at which the behavior of the matter variables changes. For instance, in the case of large accelerations $(q<-1)$, which for cosmological models means a strongly increasing expansion and for local models (like 
stars) a strongly increasing collapse, most matter variables change the sign. All matter variables display generally the same dependence on the expansion rate $H$ and are therefore of likewise importance. A more detailed discussion can only be achieved if the dependence on $\zeta^{00}$ and $h_{\alpha}^{0}$ is fixed for specified Bianchi models.

For small values of the scale factor $a$, that is, in the early cosmological phase or for objects which become very dense, the structure and the integration constants become much more important in comparison to $H$ and $q$. Besides, all matter variables show the same behavior and are therefore of equal importance. When the scale factor $a(t)$ increases, the heatflux and the anisotropic pressure essentially behave like the energy and the pressure; they dilute.

As a final remark, we would like to stress out that our results are obtained using the phenomenological approach to relativistic thermodynamics. As indicated in the introduction, in this context the vanishing entropy production (13) is a necessary condition for equilibrium, whereas it is a necessary and sufficient condition for the description of reversible processes. To consider the compatibility of these results with relativistic kinetic theory would be an interesting task for further investigations.

\section{Appendix}

\section{A. Kinematic Relations}

The following relations between the kinematic invariants are used to obtain and simplify results of the Sections 3 and 4 in kinematic terms.

$$
\begin{aligned}
\dot{u}_{\| \gamma}^{\gamma}= & \frac{1}{3}\left(\left(\dot{\Theta}+\Theta^{2}\right)\left(\zeta^{00}+1\right)+T \Theta \widehat{C}^{\gamma}{ }_{\kappa \gamma} \zeta^{\kappa 0}\right), \\
\dot{u}^{2}= & \frac{1}{9} \Theta^{2}\left(\zeta^{00}+1\right)=\frac{1}{9} \Theta^{2} h^{00}, \\
\dot{u}_{\alpha \| \beta}= & \frac{1}{3} \dot{\Theta} h_{\alpha}^{0} \delta_{\beta}^{0}+\dot{u}^{2}\left(h_{\alpha \beta}+u_{\alpha} u_{\beta}\right) \\
& +\frac{1}{3} \Theta\left(u_{\alpha} \dot{u}_{\beta}+\omega_{\alpha \beta}\right)-\dot{u}_{\alpha} \dot{u}_{\beta} \\
& -\omega_{\kappa(\alpha} u_{\beta)} \dot{u}^{\kappa}-\frac{1}{2} T \dot{u}^{\kappa} \widehat{C}^{\mu}{ }_{\kappa(\alpha} h_{\beta) \mu}, \\
\omega^{\tau \mu}{ }_{\| \mu} h_{\alpha \tau}= & \omega_{\alpha \mu} \dot{u}^{\mu}-\frac{1}{2} T \omega_{\gamma \kappa} \widehat{C}_{\mu \sigma}^{\tau} \zeta^{\mu \kappa} \zeta^{\nu \sigma} h_{\alpha \tau} \\
& +T \omega_{\alpha \kappa} \widehat{C}_{\mu \rho}^{\rho} \zeta^{\mu \kappa} .
\end{aligned}
$$

\section{B. Tetrad Formulation of Curvature}

The connection coefficients in the tetrad formulation (see Section 2), the so-called Ricci rotation coefficients, can be expressed in terms of the Christoffel symbols $\Gamma_{c}{ }^{d}$,

$$
\Omega_{c}^{\mu}{ }_{\nu}=\theta_{d}^{\mu}{ }_{d}{ }_{v}^{b} \Gamma_{c b}^{d}-\theta_{v}^{b} \theta_{b, c}^{\mu}
$$

or, due to (18) by the structure constants (21), respectively,

$$
\begin{aligned}
\Omega_{\rho}{ }^{\mu}{ }_{\nu}= & \frac{\dot{a}}{a}\left(\delta_{\rho}^{\mu} \delta_{\nu}^{0}-\zeta_{\nu \rho} \zeta^{\mu 0}\right) \\
& +\frac{1}{2 a} \zeta^{\kappa \mu}\left(\widehat{C}^{\gamma}{ }_{\nu \rho} \zeta_{\kappa \gamma}+\widehat{C}^{\gamma}{ }_{\rho \kappa} \zeta_{\nu \gamma}-\widehat{C}^{\gamma}{ }_{\kappa \nu} \zeta_{\rho \gamma}\right) .
\end{aligned}
$$

Determining the Riemannian curvature tensor by the Ricciidentity and the tetrads,

$$
R_{m b c d} \theta_{\alpha}{ }^{m}=\theta_{\alpha b ; c ; d}-\theta_{\alpha b ; d ; c},
$$

the Ricci tensor can be brought to the form

$$
\begin{aligned}
R_{\alpha \beta} & \\
= & -\left(\frac{\dot{a}}{a}\right)_{, 0}\left(2 \delta_{\alpha}^{0} \delta_{\beta}^{0}+\zeta^{00} \zeta_{\alpha \beta}\right)-3\left(\frac{\dot{a}}{a}\right)^{2} \zeta^{00} \zeta_{\alpha \beta} \\
& -\frac{\dot{a}}{a^{2}}\left(\widehat{C}^{\gamma}{ }_{\kappa \beta} \zeta^{\kappa 0} \zeta_{\alpha \gamma}+\widehat{C}^{\gamma}{ }_{\kappa \alpha} \zeta^{\kappa 0} \zeta_{\beta \gamma}+\widehat{C}^{\rho}{ }_{\mu \rho} \zeta^{\mu 0} \zeta_{\alpha \beta}\right) \\
& -\frac{1}{2 a^{2}} \widetilde{R}_{\alpha \beta}
\end{aligned}
$$

with

$$
\begin{aligned}
\widetilde{R}_{\alpha \beta} & \\
= & -\frac{1}{2}\left(-\widehat{C}^{\gamma}{ }{ }_{\beta} \widehat{C}_{\mu \rho}^{\rho} \zeta_{\alpha \gamma} \zeta^{\mu \kappa}+\widehat{C}^{\mu}{ }_{\nu \beta} \widehat{C}^{\nu}{ }_{\alpha \mu}\right. \\
& -\widehat{C}^{\gamma}{ }_{\kappa \alpha} \widehat{C}_{\mu \rho}^{\rho} \zeta_{\beta \gamma} \zeta^{\mu \kappa} \\
& \left.-\widehat{C}^{\mu}{ }_{\nu \beta} \widehat{C}^{\tau}{ }_{\sigma \alpha} \zeta_{\mu \tau} \zeta^{\nu \sigma}+\frac{1}{2} \widehat{C}^{\gamma}{ }^{\gamma}{ }{ }^{\tau}{ }_{\mu \sigma} \zeta_{\beta \gamma} \zeta^{\mu \kappa} \zeta_{\alpha \tau} \zeta^{\nu \sigma}\right) .
\end{aligned}
$$

Accordingly, the Ricci scalar becomes

$$
\begin{aligned}
R= & -6\left(\frac{\dot{a}}{\mathrm{a}}\right)_{, 0} \zeta^{00}-12\left(\frac{\dot{a}}{a}\right)^{2} \zeta^{00}-6 \frac{\dot{a}}{a^{2}} \widehat{C}_{\mu \rho}^{\rho} \zeta^{\mu 0} \\
& -\frac{1}{a^{2}} \widetilde{R}
\end{aligned}
$$

with

$$
\begin{aligned}
\widetilde{R}= & \widehat{C}^{\gamma}{ }_{\kappa \gamma} \widehat{C}_{\mu \rho}^{\rho} \zeta^{\mu \kappa}-\frac{1}{2} \widehat{C}^{\mu}{ }_{\nu \beta} \widehat{C}^{\nu}{ }_{\alpha \mu} \zeta^{\alpha \beta} \\
& +\frac{1}{4} \widehat{C}^{\mu}{ }_{\nu \beta} \widehat{C}^{\tau}{ }_{\sigma \alpha} \zeta^{\nu \sigma} \zeta_{\mu \tau} \zeta^{\alpha \beta} .
\end{aligned}
$$

The expressions $\widetilde{R}_{\widehat{\alpha} \widehat{\beta}}$ of (B.5) and equivalently $\widetilde{R}$ of (B.7) can be identified with the Ricci tensor and the Ricci scalar of 3dimensional Bianchi spaces [45].

This results in the following shape of the Einstein tensor:

$$
\begin{aligned}
G_{\alpha \beta}= & R_{\alpha \beta}-\frac{1}{2} R \zeta_{\alpha \beta} \\
= & 2\left(\frac{\ddot{a}-\dot{a}^{2}}{a^{2}}\right)\left(\zeta_{\alpha \beta} \zeta^{00}-\delta_{\alpha}^{0} \delta_{\beta}^{0}\right)+3\left(\frac{\dot{a}}{a}\right)^{2} \zeta_{\alpha \beta} \zeta^{00} \\
& +2 \frac{\dot{a}}{a^{2}} \widehat{C}^{\rho}{ }_{\kappa \mu} \zeta^{\kappa 0}\left(\delta_{\rho}^{\mu} \zeta_{\alpha \beta}-\delta_{(\alpha}^{\mu} \zeta_{\beta) \rho}\right) \\
& +\frac{1}{a^{2}}\left(-\widetilde{R}_{\alpha \beta}+\frac{1}{2} \widetilde{R} \zeta_{\alpha \beta}\right) .
\end{aligned}
$$




\section{Constants of Integration}

From the field equations, $G_{\alpha \beta}=T_{\alpha \beta}$, in terms of the tetrad formulation from Section 2 and together with (5), one finds

$$
-\widetilde{R}_{\alpha \beta}+\frac{1}{2} \widetilde{R} \zeta_{\alpha \beta}={ }_{E I} c_{\alpha \beta} .
$$

Then, because of (50) the constants of integration, that is, ${ }_{\rho}{ }^{c},{ }_{p} c, q_{\alpha}^{c}$, and ${ }_{\pi} c_{\alpha \beta}$, for the energy density, the isotropic pressure, the heat-flux, and the anisotropic pressure (43)(46), become, in this order,

$$
\begin{aligned}
\rho^{c} & ={ }_{E I} c_{\alpha \beta} u^{\alpha} u^{\beta}=-\frac{1}{2} \widetilde{R}+2\left(\frac{\omega}{{ }_{T} c T}\right)^{2}, \\
{ }_{p} c= & \frac{1}{3}{ }_{E I} c_{\alpha \beta} h^{\alpha \beta}=\frac{1}{6} \widetilde{R}+\frac{2}{3}\left(\frac{\omega}{{ }_{T} c T}\right)^{2}, \\
{ }_{q} c_{\alpha}= & -{ }_{E I} c_{\beta \gamma} u^{\beta} h_{\alpha}^{\gamma}=\frac{1}{{ }_{T} c^{2} T^{2}}\left(\omega^{\kappa \mu}{ }_{\| \mu} h_{\alpha \kappa}-\omega_{\alpha \mu} \dot{u}^{\mu}\right), \\
{ }_{\pi} c_{\alpha \beta}= & { }_{E I} c_{\gamma \delta} h_{\alpha}^{\gamma} h_{\beta}^{\delta}-{ }_{p} c h_{\alpha \beta} \\
= & -\widetilde{R}_{\alpha \beta}+\frac{1}{3} \widetilde{R} h_{\alpha \beta}-\frac{2}{3}\left(\frac{\omega}{{ }_{T} c T}\right)^{2}\left(3 u_{\alpha} u_{\beta}+h_{\alpha \beta}\right) \\
& -\frac{2}{{ }_{T} c^{2} T^{2}}\left(\omega_{\rho(\alpha} u_{\beta)} \dot{u}^{\rho}+\omega^{\tau \mu}{ }_{\| \mu} h_{\tau(\alpha} u_{\beta)}\right),
\end{aligned}
$$

where in (C.4) and (C.5) it was made use of relation (A.4). If one reinserts the constants of integration (C.2)-(C.5) into the matter equations (43)-(46), they take the purely kinematic forms (51)-(54).

The summarized constants of integration, ${ }_{\rho} c,{ }_{p} c,{ }_{q} c_{\alpha}$, and ${ }_{\pi} c_{\alpha \beta}$, in this paper are pieced together as follows:

$$
\begin{aligned}
\rho^{c} & :=-{ }_{\rho} \widetilde{c}-2 c_{1}\left(2 \zeta^{00}-1\right)-2 c_{2}\left(\zeta^{00}+1\right) \\
& -2 c_{3} \widehat{C}^{\gamma}{ }_{\kappa \gamma} \zeta^{\kappa 0} \\
p & :=-{ }_{p} \widetilde{c}-\frac{2}{3} c_{2}\left(1-2 \zeta^{00}\right)+\frac{2}{3} c_{1}\left(7 \zeta^{00}+1\right)+\frac{4}{3} \\
& \cdot c_{3} \widehat{C}^{\gamma}{ }_{\kappa \gamma} \zeta^{\kappa 0}, \\
{ }_{q} c_{\alpha} & :=-{ }_{q} \widetilde{c}_{\gamma}\left(h_{\alpha}^{\gamma}-h_{\alpha}^{0} u^{\gamma}\right)-2\left(c_{1}+c_{2}\right) h_{\alpha}^{0} \\
& -c_{3} \widehat{C}_{\alpha \sigma}^{\gamma} \zeta_{\gamma 0} \zeta^{\sigma 0}, \\
{ }_{\pi} c_{\alpha \beta} & :=\frac{1}{3}\left(-{ }_{\pi} \widetilde{c}_{\gamma \delta}\left(h_{\alpha}^{\gamma}-h_{\alpha}^{0} u^{\gamma}\right)\left(h_{\beta}^{\delta}-h_{\beta}^{0} u^{\delta}\right)\right. \\
& -2\left(c_{1}-c_{2}\right)\left(h_{\alpha \beta}\left(\zeta^{00}+1\right)-3 h_{\alpha}^{0} h_{\beta}^{0}\right) \\
& \left.+2 c_{3} \widehat{C}_{\mu \kappa}^{\rho} \zeta^{\kappa 0}\left(\delta_{\rho}^{\mu} h_{\alpha \beta}-3 \delta_{(\alpha}^{\mu} h_{\beta) \rho}\right)\right) .
\end{aligned}
$$

The occurring objects $c_{1}, c_{2}, c_{3},{ }_{\rho} \widetilde{c},{ }_{p} \widetilde{c},{ }_{q} \widetilde{c}_{\widehat{\alpha}}$, and ${ }_{\pi} \widetilde{c}_{\widehat{\alpha} \widehat{\beta}}$ are the actual constants of integration yielded by the following integrals, which are to be calculated in Section 3.1:

$$
\begin{aligned}
& \int \ddot{a} \dot{a} d x^{0}=\frac{\dot{a}^{2}}{2}+c_{1}, \\
& \int \ddot{a} a d x^{0}=\ddot{a} a-\frac{\dot{a}^{2}}{2}+c_{2}, \\
& \int \ddot{a} d x^{0}=\dot{a}+c_{3}, \\
& \int\left(a^{2} \rho\right)_{, 0} d x^{0}=a^{2} \rho+{ }_{\rho} \widetilde{c}, \\
& \int\left(a^{2} p\right)_{, 0} d x^{0}=a^{2} p+{ }_{p} \widetilde{c}, \\
& \int\left(a^{2} q_{\alpha}\right)_{, 0} d x^{0}=a^{2} q_{\alpha}+{ }_{q} \tilde{c}_{\beta}\left(h_{\alpha}^{\gamma}-h_{\alpha}^{0} u^{\gamma}\right), \\
& \int 3\left(a^{2} \pi_{\alpha \beta}\right)_{, 0} d x^{0} \\
& \quad=a^{2} \pi_{\alpha \beta}+{ }_{\pi} \tilde{c}_{\gamma \delta}\left(h_{\alpha}^{\gamma}-h_{\alpha}^{0} u^{\gamma}\right)\left(h_{\beta}^{\delta}-h_{\beta}^{0} u^{\delta}\right) .
\end{aligned}
$$

\section{Competing Interests}

The authors declare that there is no conflict of interests regarding the publication of this paper.

\section{References}

[1] G. F. R. Ellis, "Relativistic cosmology," in Proceedings of the International School of Physics "Enrico Fermi", Course 47: General Relativity and Cosmology, R. K. Sachs, Ed., pp. 104-182, Academic Press, New York, NY, USA, 1971.

[2] V. A. Korotky and Y. N. Obukhov, "Bianchi-II rotating world," Astrophysics and Space Science, vol. 260, no. 4, pp. 425-439, 1998.

[3] Y. N. Obukhov, "Observations in rotating cosmologies. Gauge theories of fundamental interactions," in Proceedings of the 32nd Semester in the Stefan Banach International Mathematical Center, M. Pawlowski and R. Raczka, Eds., World Scientific, Warsaw, Poland, 1990.

[4] Y. N. Obukhov, "On physical foundations and observational effects of cosmic rotation," in Colloquium on Cosmic Rotation, M. Scherfner, Ed., Wissenschaft und Technik, 2000.

[5] Y. N. Obukhov, T. Chrobok, and M. Scherfner, "Shear-free rotating inflation," Physical Review D, vol. 66, no. 4, Article ID 043518, 2002.

[6] S. Capozziello, V. F. Cardone, E. Elizalde, S. Nojiri, and S. D. Odintsov, "Observational constraints on dark energy with generalized equations of state," Physical Review D-Particles, Fields, Gravitation and Cosmology, vol. 73, no. 4, Article ID 043512, 2006.

[7] R. Myrzakulov and L. Sebastiani, "Bounce solutions in viscous fluid cosmology," Astrophysics and Space Science, vol. 352, no. 1, pp. 281-288, 2014.

[8] S. Nojiri and S. D. Odintsov, "Final state and thermodynamics of a dark energy universe," Physical Review D, vol. 70, no. 10, Article ID 103522, 2004. 
[9] S. Nojiri and S. D. Odintsov, "Inhomogeneous equation of state of the universe: phantom era, future singularity, and crossing the phantom barrier," Physical Review D, vol. 72, Article ID 023003, 2005.

[10] R. Treciokas and G. F. R. Ellis, "Isotropic solutions of the Einstein-Boltzmann equations," Communications in Mathematical Physics, vol. 23, no. 1, pp. 1-22, 1971.

[11] T. Chrobok and H.-H. V. Borzeszkowski, "Thermodynamic equilibrium and rotating space-time," in Gödel-Type Spacetimes: History and New Developments, M. Scherfner and M. Plaue, Eds., pp. 7-22, Kurt Gödel Society, Collegium Logicum X, 2010.

[12] J. Ehlers, P. Geren, and R. K. Sachs, "Isotropic solutions of the Einstein-Liouville equations," Journal of Mathematical Physics, vol. 9, no. 9, pp. 1344-1349, 1968.

[13] K. L. Duggal, "Relativistic fluids with shear and timelike conformal collineations," Journal of Mathematical Physics, vol. 28, no. 11, pp. 2700-2704, 1987.

[14] D. R. Oliver Jr. and W. R. Davis, "On certain timelike symmetry properties and the evolution of matter field space-times that admit them," General Relativity and Gravitation, vol. 8, no. 11, pp. 905-914, 1977.

[15] W. Hasse and V. Perlick, "Geometrical and kinematical characterization of parallax-free world models," Journal of Mathematical Physics, vol. 29, no. 9, pp. 2064-2068, 1988.

[16] A. A. Coley, "Fluid spacetimes admitting a conformal Killing vector parallel to the velocity vector," Classical and Quantum Gravity, vol. 8, no. 5, pp. 955-968, 1991.

[17] R. K. Barrett and C. A. Clarkson, "Undermining the cosmological principle: almost isotropic observations in inhomogeneous cosmologies," Classical and Quantum Gravity, vol. 17, no. 24, pp. 5047-5078, 2000.

[18] C. A. Clarkson and R. K. Barrett, "Does the isotropy of the CMB imply a homogeneous universe? Some generalized EGS theorems," Classical and Quantum Gravity, vol. 16, no. 12, pp. 3781-3794, 1999.

[19] C. A. Clarkson, A. A. Coley, E. S. D. O’Neill, R. A. Sussman, and R. K. Barrett, "Inhomogeneous cosmologies, the copernican principle and the cosmic microwave background: more on the EGS theorem," General Relativity and Gravitation, vol. 35, no. 6, pp. 969-990, 2003.

[20] H. Stephani, D. Kramer, M. MacCallum, C. Hoenselaers, and E. Herlt, Exact Solutions of Einstein's Field Equations, Cambridge Monographs on Mathematical Physics, Cambridge University Press, 2nd edition, 2003.

[21] T. Chrobok and H.-H. v. Borzeszkowski, "Thermodynamical equilibrium and spacetime geometry," General Relativity and Gravitation, vol. 38, no. 3, pp. 397-415, 2006.

[22] C. Eckart, "The thermodynamics of irreversible processes. III. Relativistic theory of the simple fluid," APS Journals Archive, vol. 58, no. 10, p. 919, 1940.

[23] W. Israel, "Covariant fluid mechanics and thermodynamics: an introduction," in Relativistic Fluid Dynamics, A. Anile and Y. Choquet-Bruhat, Eds., vol. 1385 of Lecture Notes in Mathematics, pp. 152-210, Springer, Berlin, Germany, 1989.

[24] G. Neugebauer, Relativistische Thermodynamik, Akademie, 1980.

[25] S. Weinberg, Gravitation and Cosmology: Principles and Applications of the General Theory of Relativity, John Wiley \& Sons, New York, NY, USA, 1972.

[26] H.-H. Borzeszkowski, T. Chrobok, and W. Muschik, "Thermodynamical equilibrium and space-time geometry-a survey,"
Communications in Applied and Industrial Mathematics, vol. 1, no. 2, pp. 206-215, 2010.

[27] G. O. Schellstede, H.-H. von Borzeszkowski, T. Chrobok, and W. Muschik, "The relation between relativistic and nonrelativistic continuum thermodynamics," General Relativity and Gravitation, vol. 46, article 1640, 2014.

[28] H. Stephani, General Relativity, Cambridge University Press, 1982.

[29] M. L. Bedran and M. O. Calvao, "Reversibility and spacetime symmetries," Classical and Quantum Gravity, vol. 10, no. 4, pp. 767-771, 1993.

[30] J. Triginer and D. Pavón, "On the thermodynamics of tilted and collisionless gases in Friedmann-Robertson-Walker spacetimes," Classical and Quantum Gravity, vol. 12, no. 1, pp. 199-207, 1995.

[31] W. Zimdahl and A. B. Balakin, "Thermodynamic equilibrium in the expanding universe," General Relativity and Gravitation, vol. 31, no. 9, pp. 1395-1405, 1999.

[32] W. Zimdahl and A. B. Balakin, "Cosmological thermodynamics and deflationary gas universe," Physical Review D, vol. 63, no. 2, Article ID 023507, 2000.

[33] W. Zimdahl, D. J. Schwarz, A. B. Balakin, and D. Pavón, “Cosmic antifriction and accelerated expansion," Physical Review D, vol. 64, no. 6, Article ID 063501, 2001.

[34] W. Zimdahl, D. J. Schwarz, A. B. Balakin, and D. Pavon, "Conformal symmetry and cosmological entropy production," Entropy, vol. 4, no. 3, pp. 49-127, 2002.

[35] A. R. King and G. F. Ellis, "Tilted homogeneous cosmological models," Communications in Mathematical Physics, vol. 31, pp. 209-242, 1973.

[36] S. Chandrasekhar, The Mathematical Theory of Black Holes, Clarendon Pr. u.a, Oxford, UK, 1983.

[37] A. A. Coley and D. J. McManus, "On spacetimes admitting shear-free, irrotational, geodesic time-like congruences," Classical and Quantum Gravity, vol. 11, no. 5, pp. 1261-1282, 1994.

[38] V. Perlick, "Gravitational lensing from a spacetime perspective," Living Reviews in Relativity, vol. 7, article 9, 2004.

[39] E. Rebhan, Theoretische Physik: Relativitätstheorie und Kosmologie, Springer Spektrum, Heidelberg, Germany, 2012.

[40] T. Chrobok, Scherungsfreie fluide in der allgemeinen relativitätstheorie [Ph.D. thesis], TU, Berlin, Germany, 2004.

[41] S. Harris, "Conformally stationary spacetimes," Classical and Quantum Gravity, vol. 9, no. 7, pp. 1823-1827, 1992.

[42] L. Herrera, A. Di Prisco, and J. Ibáñez, "Reversible dissipative processes, conformal motions and Landau damping," Physics Letters A, vol. 376, no. 8-9, pp. 899-900, 2012.

[43] I. Müller and T. Ruggeri, Extended Thermodynamics, vol. 37, Springer, New York, NY, USA, 1993.

[44] A. Kashlinsky, F. Atrio-Barandela, and H. Ebeling, "Measuring bulk motion of X-ray clusters via the kinematic SunyaevZeldovich effect: summarizing the 'dark flow' evidence and its implications," https://arxiv.org/abs/1202.0717.

[45] R. M. Wald, General Relativity, University of Chicago Press, Chicago, Ill, USA, 1984. 

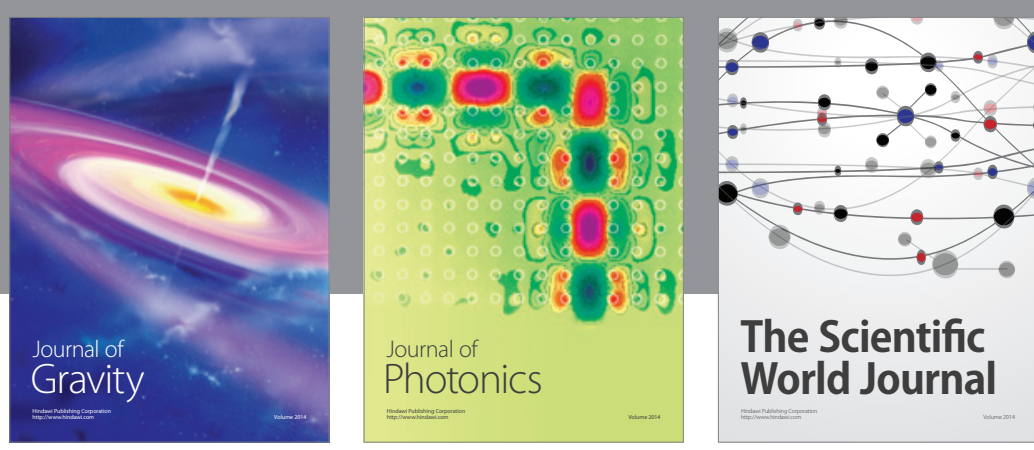

The Scientific World Journal
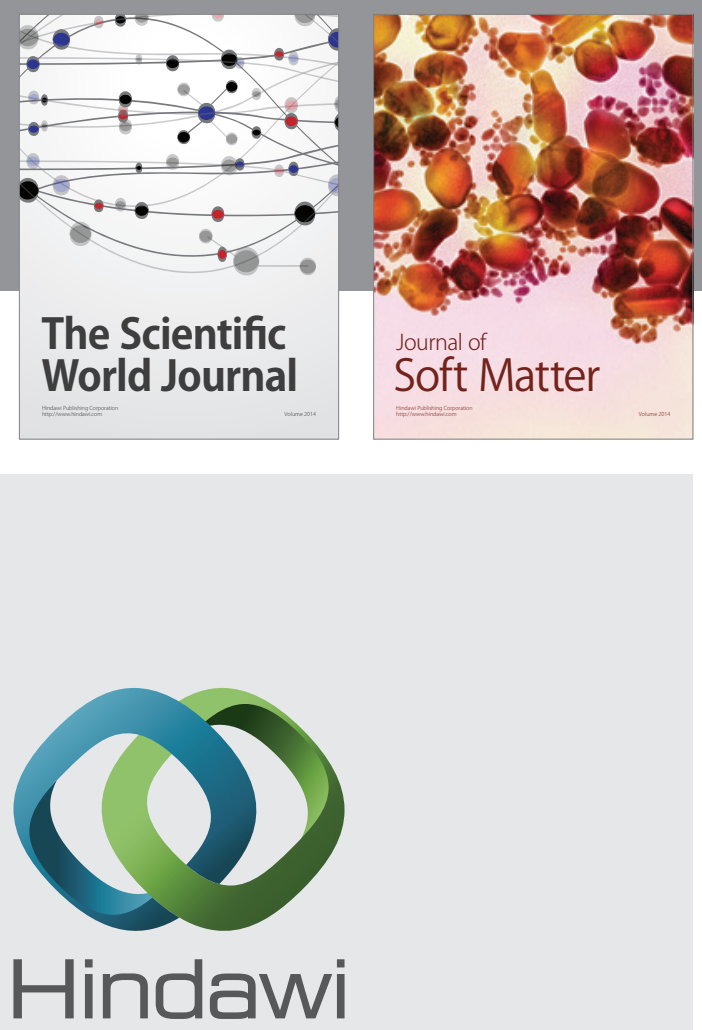

Submit your manuscripts at

http://www.hindawi.com

nternational Journal of

Statistical Mechanics
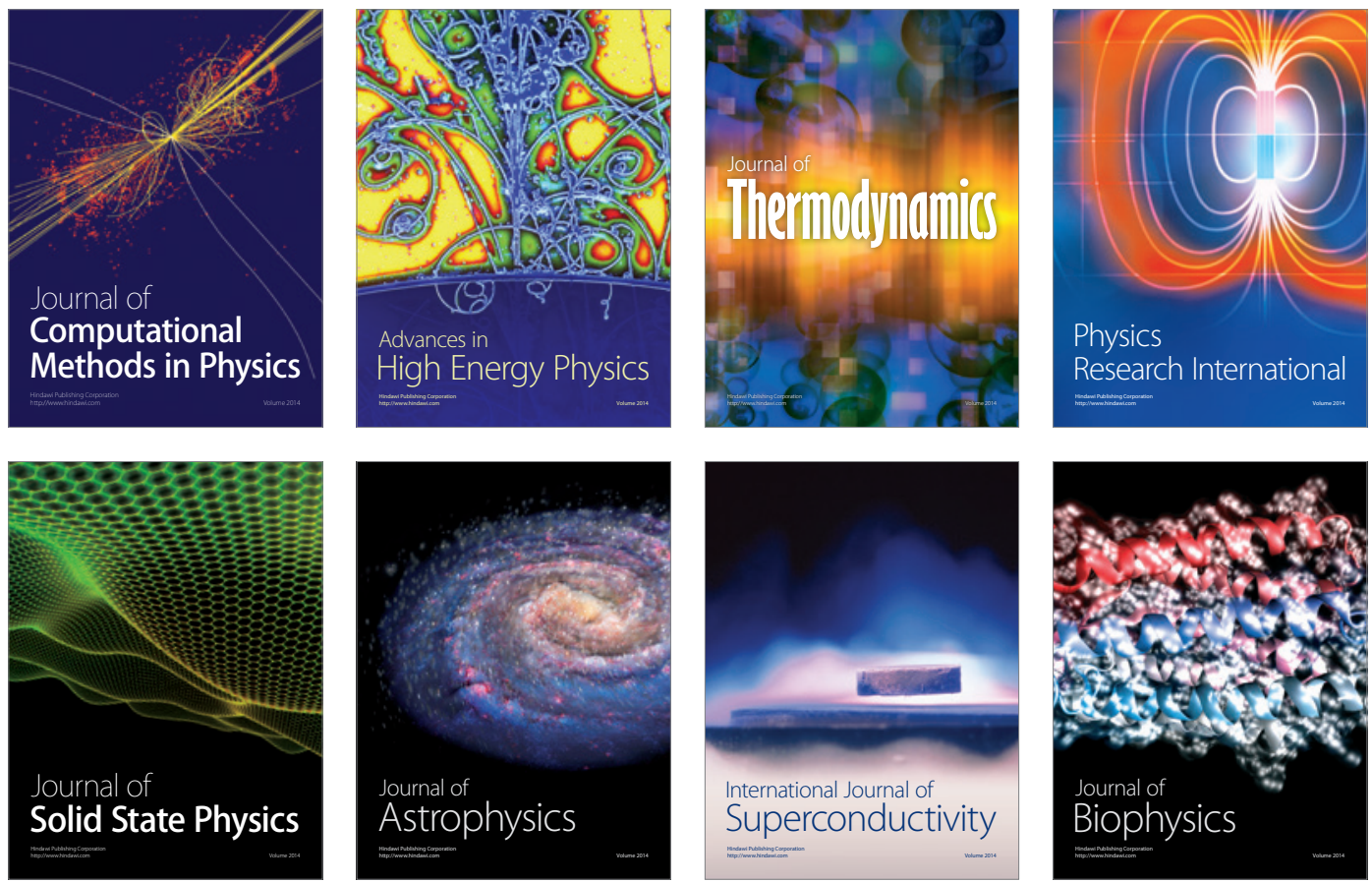
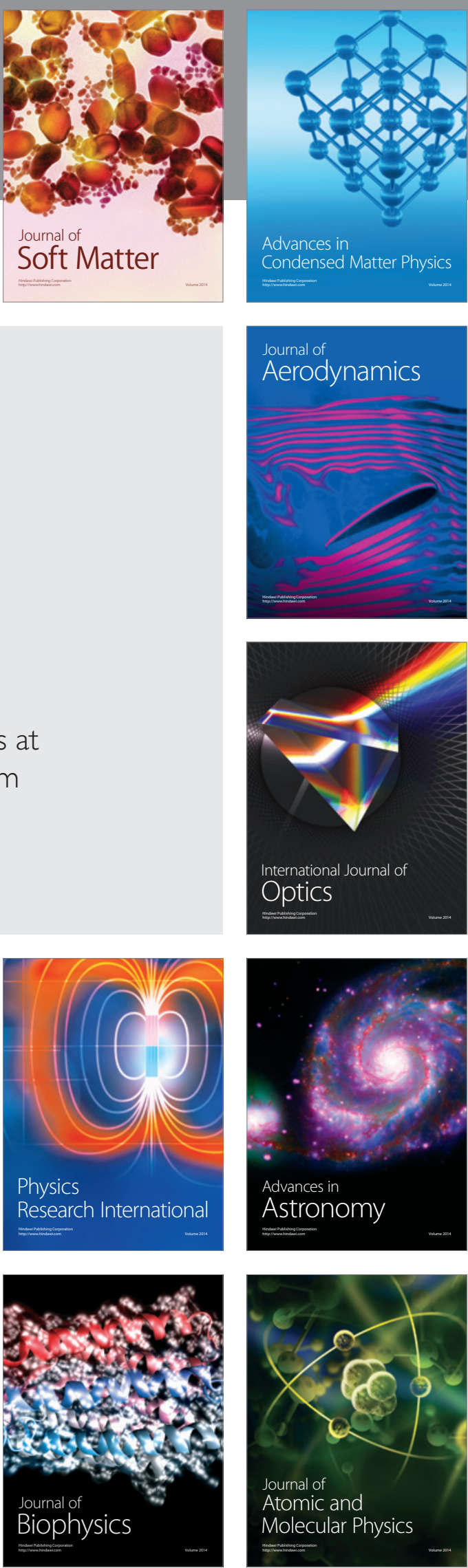